\title{
Integrating IoT Modern Communication Architectures into the New Generation of VR/MR Environments
}

\author{
Dragos Sebastian CRISTEA ${ }^{\star}$, Ruben Cantarero NAVARRO ${ }^{\star \star}$, Javier Sánchez RIQUELME ${ }^{\star \star \star}$, \\ Marius IVANOV ${ }^{\star \star \star \star}$, Muneeb ANWAR ${ }^{\star \star \star \star \star}$, George SUCIU ${ }^{\star \star \star \star \star \star}$
}

\begin{tabular}{l} 
A R T I C L E I N F O \\
\hline Article history: \\
Accepted August 2019 \\
Available online August 2019 \\
\hline JEL Classification \\
C88, L86 \\
Keywords: \\
Internet of Things, Mixed Reality, \\
Virtual Reality, Smart City, \\
Evacuation, VR environment, \\
HoloLens, weather sensors
\end{tabular}

Over the last decade, applications based on mobile devices, sensors, and actuators have grown smarter, empowering communication between devices and the achievement of more complex tasks. In 2008 the number of connected devices exceeded the global population [13], and the amount keeps rising exponentially until today. Smartphones, embedded systems, wireless sensors, and practically every electronic device are connected to a local network or the internet, reaching to the era of the Internet of Things (IoT). Increase in the number of devices sums up to increase in the amount of data collected by those devices as well. New applications appear that interpret the collected data to make significant correlations and possible conclusions, leading to Artificial Intelligence (AI) via Machine Learning (ML) algorithms.

In IoT terms, each connected device is considered a thing. Things generally consist of physical sensors, actuators, and an implanted system with a microprocessor. Things need to communicate with each other, generating the need for Machine-to-Machine (M2M) communication. The communication can be short distance using wireless technologies such as Wi-Fi, Bluetooth, and ZigBee, or wide-range using mobile networks such as WiMAX, LoRa, Sigfox, CAT M1, NB-IoT, GSM, GPRS, 3G, 4G, LTE, and 5G [15]. IoT applications have already developed in many aspects, the so-called smart city. We could list the most critical applications in the following categories [14]:

- Smart Homes: This section comprises of home devices, such as fridges, washing machines, or light bulbs, that have been developed and are capable of communicating with each other or with authorized users via the internet, giving better monitoring and management of the devices as well as energy consumption optimization. Apart from traditional devices, new technologies expanded, presenting smart home assistants, smart door locks, etc. ${ }^{\star}$ Dunarea de Jos University of Galati, Romania, ${ }^{\star \star}$ University of Castilla - La Mancha, Spain, ${ }^{\star \star \star}$ Answaretech, Murcia, Spain, ${ }^{\star \star \star \star}$ Altfactor, Galati, Romania, ${ }^{\star \star \star \star \star,},{ }^{\star \star \star \star \star \star}$ Beia,
Bucharest, Romania, E-mail addresses: dragoscristea@yahoo.com (D. S. Cristea - Corresponding author), ruben.cantarero@uclm.es (R. C. Cantarero), jsanchez@answare-tech.com (J. S. Riquelme), marius.ivanov@altfactor.ro (M. Ivanov), ma@beia.ro (M. Anwar), george.suciu@beia.ro (G. Suciu) 
- Health-care assistance: New instruments have been exploited to improve a patient's well-being. Plasters with wireless sensors can monitor a wound's situation and report the data to the doctor without the requirement for their physical appearance. Additional sensors in the form of wearable devices or small implants can trace and inform a wide assortment of measurements, such as heart rate, blood oxygen level, blood sugar level, or temperature.

- Smart Transportation: Using sensors installed to the vehicles, or mobile devices and devices installed in the city, it is permissible to propose optimized route instructions, accessible parking reservations, energy-efficient street lighting, telematics for public medians of transportation, accident avoidance, and autonomous driving.

- Environmental Conditions Monitoring: Wireless sensors dispersed in the city make proper infrastructure for a broad kind of environmental conditions monitoring. Barometers, humidity sensors, or ultrasonic wind sensors can benefit from generating advanced weather stations. Moreover, smart sensors can monitor the air quality and water pollution levels over the city.

The phrase Virtual Reality (VR) relates to a computer technology that resembles the physical appearance of a user in artificially-generated circumstances. VR allows performing in real time, a certain number of actions illustrated by one or more computer programs and, therefore, to encounter a certain amount of visual, auditory, or touch sensations [16]. From a particular viewpoint, virtual reality and the Internet of Things are two of the most influential technologies to emerge in the past decade or more. VR can genuinely transform the world in remarkable, surprising ways, while the Internet of Things already has improved the means we live our lives. It is the confluence of these two developments, though, that gives the most hope and possibility of all.

At this level of competition, it is transparent to approximately everyone in tech that both VR and IoT are distant from a flash in the pan. They have both obtained the public vision as well as demonstrated their deserving in the industrial sector, and they are both swiftly becoming enmeshed in modern society.

Therefore, it is not unusual anymore that the corporates and SMEs have already made huge investments into the expansion of new applications of both virtual reality and the Internet of Things. In 2016, an approximated $\$ 1.1$ billion was funded in AR and VR [19,20,5]. A few states of the art studies proposing to meet the whole VR domain exists. A recent generalized summary is provided by Hale and Stanney [6] also providing details and solutions in the different application fields. The current research defining VR development is scarce and reliable resources are few. Details on the first versions of the Oculus Rift are outlined in a book by Davies [2]. The VR Book by Jerald [8] attempts to center on both technology and HCI. A hands-on guide for the development of VR applications has latterly been written by Toni Parisi [12]. More recent ones are rendered by Muhanna [10] who also introduces a taxonomy covering the several types of VR. He concentrates on the academic and industrial domain rather than the recent developments in consumer VR and puts influential importance on CAVE-like systems. They stated that VR implementation could be employed to overcome inconsistency and assess numerous alternative solutions. Similarly, Dávideková et al. [1], studied various learning procedures and discovered the limitations and insufficiencies of currently used conventional teaching methods. In [18], the authors proposed a new criterion: The Virtual Environment of Things (VEoT). Their proposal points at integrating real-world objects and virtual-world objects/avatars. To authorize the VEoT prototype, a VEoT-based NVE (Networked Virtual Environment), called X-Campus has been implemented. The purpose of X-Campus was to lead users to navigate in the building of the National Central University. In [7], the authors created a prototype of a smart campus based on IoT and VR. For instance, in [17], the authors examined the efficiency and the requirement of using IoT in SCC (Smart College Campus); moreover, they engaged in the matter of using IoT for a smart campus application, where the lecture room had access to elearning applications with the support of IoT tools. The work in [9] introduced a virtual lab based on VR where students can perform the virtual manufacture of instruments. VR is also used to design interactive 3D virtual laboratories to learn chemistry [11]. In [3], VR was used to create a 3D environment to guide students the unique relativity concept. In [4], the authors illustrated a review of diverse practices related to the use of VR in dental education.

Mixed reality represents a combination of physical and virtual worlds that holds both real and computer-generated objects. The two worlds are "mixed" mutually to form a realistic environment. A user can navigate this environment and communicate with both real and virtual objects. Mixed reality (MR) merges features of virtual reality (VR) and augmented reality (AR). It is sometimes called "enhanced" AR since it is related to AR technology, but contributes more physical interaction. The current release of Magic Leap (ML) has been creating a lot of curiosity in MR, but the technology itself has been around for a while - Microsoft launched HoloLens in 2016. Though over time, the selection of mixed reality technology will vanish, and we will be left with the most essential bit of the content. In practice, quality control administrators will wear Microsoft HoloLens smart glasses in which all the digitalized engine components will be integrated. Through the glasses and Mixed Reality interface, operators will see decision-making guidance that will supervise them through the most complex control operations. At the moment, operators managing on control points are still using paper instructions." This is a positive step forward with considerations to ease of operations [21]. 
In this paper we are going to highlight how our IoT architecture is integrated in two MR/VR study cases.

\section{IoT Communication Architecture}

The IoT architecture comprises a set of basic interfaces to communicate a value (on/off and analog values) and a set of properties of that value (e.g. position in mobile sensors). The serialization / deserialization and transmission of the interface invocation is done following a Remote Procedure Call philosophy using the Internet Communication Engine Protocol [1] (IceP). IceP is an open binary protocol used in the middleware Internet Communication Engine [1] (Ice). This implementation enables the use of LPWAN technologies with a limited Maximum Transmission Unit and a seamless integration with the rest of the middleware infrastructure. In this way, for example, the method invocation is injected directly in the Icestorm Service (the broker event service of Ice) and distributed to other services subscribed to the topic where event is generated (e.g. all power sensors send information to the energy topic). Together with these interfaces two services are used for efficient IoT information provision: The IoT architecture comprises a set of basic interfaces to communicate a value (on/off and analog values) and a set of properties of that value (e.g. position in mobile sensors). The serialization/deserialization and transmission of the interface invocation is done following a Remote Procedure Call philosophy using the Internet Communication Engine Protocol [1] (IceP). IceP is an open binary protocol used in the middleware Internet Communication Engine [1] (Ice). This implementation enables the use of LPWAN technologies with a limited Maximum Transmission Unit and a seamless integration with the rest of the middleware infrastructure. In this way, for example, the method invocation is injected directly in the Icestorm Service (the broker event service of Ice) and distributed to other services subscribed to the topic where event is generated (e.g. all power sensors send information to the energy topic). Together with these interfaces two services are used for efficient IoT information provision: The IoT architecture comprises a set of basic interfaces to communicate a value (on/off and analog values) and a set of properties of that value (e.g. position in mobile sensors). The serialization/deserialization and transmission of the interface invocations is done following a Remote Procedure Call philosophy using the Internet Communication Engine Protocol [1] (IceP). IceP is an open binary protocol used in the middleware Internet Communication Engine [1] (Ice). This implementation enables the use of LPWAN technologies with a limited Maximum Transmission Unit and a seamless integration with the rest of the middleware infrastructure. In this way, for example, the method invocation is injected directly in the Icestorm Service (the broker event service of Ice) and distributed to other services subscribed to the topic where event is generated (e.g. all power sensors send information to the "energy" topic). Together with these interfaces two services are used for efficient IoT information provision:

- The property service is a key-value chain store for accessing to the static properties of IoT nodes (e.g. static position, manufacturer, last revision, accuracy, etc.). This service avoids to ask directly to the IoT node for these static properties saving power and traffic bandwidth of LPWAN.

- The dashboard cache is devoted to provide with the last reading of each IoT sensor of a Citisim controlled area. This service enables to get a snapshot of a specific area without waiting of new readings with different update intervals. Of course, each reading in this cache has a timestamp and a valid period.

The data model is specified by an ontology which specifies the name and type of sensors, actuators, services, properties, etc. establishing a common vocabulary for developers. The physical area is modeled using Open Geospatial Consortium IndoorGML [2] standard extended with Open Location Codes [3] from Google. The IndoorGML is used to specify the coverage of sensors deployed, using a sensor layer, and the relation with the physical location using Open Location Codes.

With this approach, we can export to the virtual world developed in Unity, not only the location of an IoT device but the area of sensing/influence of sensors of that IoT device. From a practical point of view, the virtual world uses the subscription to the broker event service together with the property service and the dashboard cache for representing the physical conditions of the real world in its virtual world counterpart. The meeting point of IoT developers and MR/VR developers is a C\# library that acts as wrapper of the IoT information. This library is integrated in the Unity 3D development environment used for MR/VR service development. A config file enables the connection of the MR/VR with any CitiSim instance. The MR/VR service can get updates of the information of the real world subscribing to different topics and/or get a snapshot of the real world using the dashboard cache service.

\section{Study Case 1 - Using Mixed Reality for Indoor Emergency training in Smart Cities}

This study case presents how Mixed Reality can be applied for the development of an application based on Microsoft HoloLens $($ C headset at ANSWARETECH, by using data provided through the described IOT communication infrastructure. Mixed Reality (MR) allow users to mix real and virtual elements to create an improved context in which users can place virtual elements in a location of the real world and can interact with them using natural interactions like gestures or voice actions. The number of useful functionalities that these new devices can offer to a Smart City is huge, like infrastructures maintenance support, immersive 
forecast information, simulation and recreation of dangerous situations or accidents, real-time information from connected sensors and city services (like public transport status, traffic, city events...). The MR Application presented in this paper is focused on covering 4 topics: virtual evacuation drills, media content display, real-time sensors information access and domotic actions. In the virtual evacuation drill scenarios, by using virtual elements display capabilities of HoloLens $\mathbb{C}$, building 3D models are loaded over the real building and multiple models of persons are placed inside their rooms and halls. Using a simulation service developed by University Politecnica of Madrid (UPM), these persons are provided with a logic that simulates human behavior in-doors. The persons' logic also provides simulated behavior for emergency situations and makes each person to take a role, e.g., leader, family member, scary. All this behavior is shown through the headset. The application also has a service to display evacuation routes generated by a service developed by University of Castilla La Mancha (UCLM) to help user to come outside the building as fast as possible.

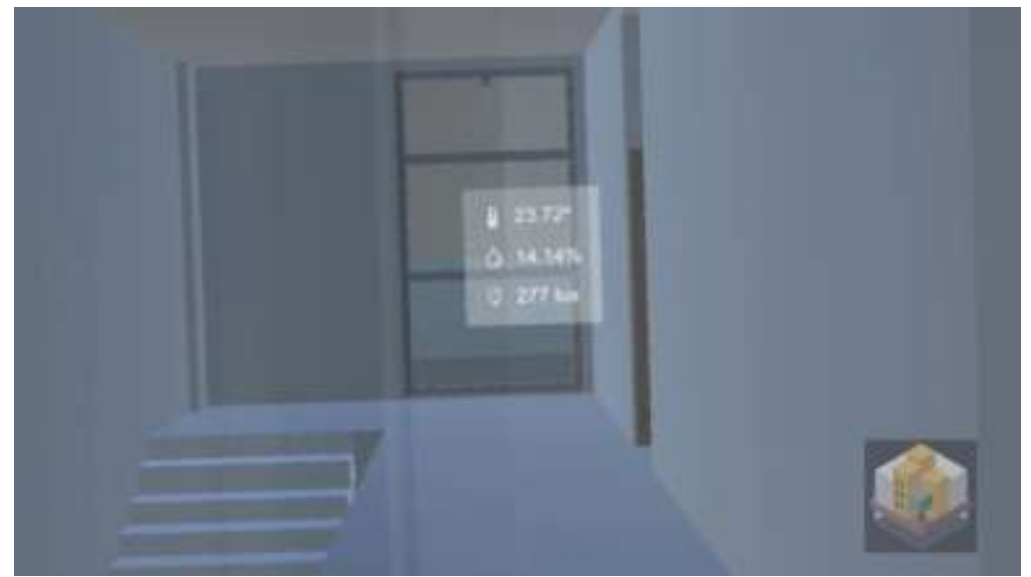

Figure 1 ITSI Building in UCLM displayed and sensor information. Shot directly from HoloLens@ MR Application

For media content display holograms are used to show videos, images, texts or presentations that could support a professional to make a more efficient job resolving doubts, accessing extra information, or just learning things in place (see figure $\mathrm{X}$ ). This media is stored in a cloud platform and serves the content to user, who can filter contents.

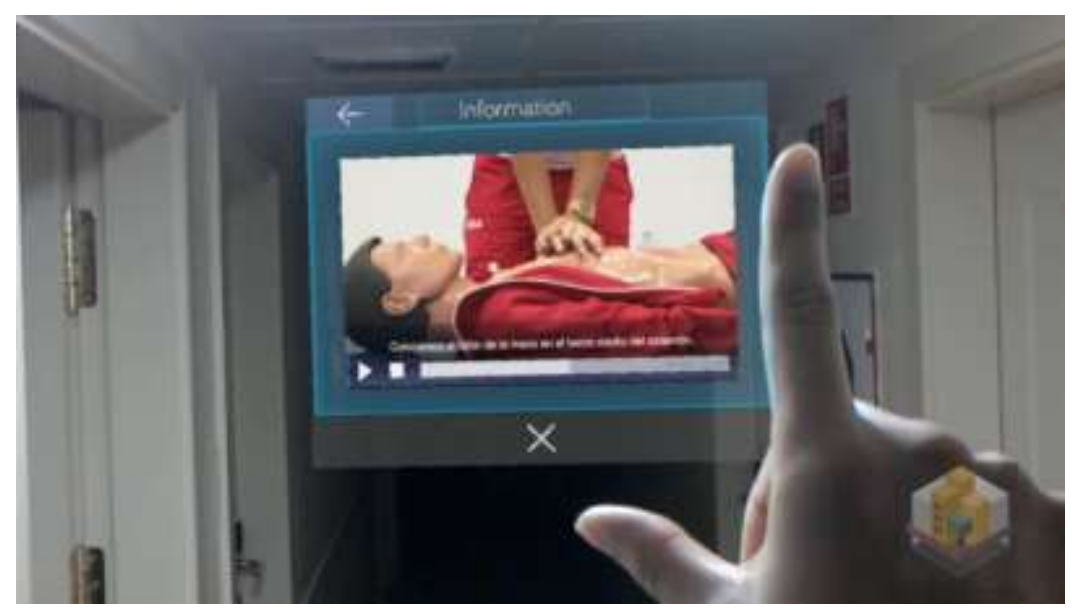

Figure 2. First-aid video shown in HoloLens@ MR Application

Real-time sensors information is integrated by using the architecture presented above. IoT adoption is growing incredibly fast since some years, cities are going to be one of the most affected by this innovation. Based on an optimized IoT communication library, a module was created to integrate all sensors information and emit it to different listeners (integrated applications). The MR Application is one of these listeners, and it uses sensors information to display interesting information about building or electrical devices to user. Domotic actions are implemented starting from the fact that natural interaction is one of the main points in Mixed Reality, and, in a Smart City context, this possibility gains value when applying it into devices management. At outside, there are tons of activities than can get benefits from in-place domotic functionalities: management of toggleable elements like fountains, streetlights or bollards, edition of texts in digital informative panels, voice message sending through public address systems, change advertisement displays, lift bridges. Inside buildings users also make their life easier with tone of functionalities: toggle 
lights and change its color or intensity, change TV channels, increase or decrease air conditioner temperature, enable or disable smart plugs. In the Mixed Reality pilot application, the domotic actions to test are lock/unlock doors and toggle lights. Emergency use-case pilot of the MR application is planned to be carried out at University Castilla a Mancha (UCLM) in Spain. A user will wear the HoloLens in an emergency simulated situation and will navigate through the building, following a generated evacuation route. While walking, information of sensors coming from different rooms will be shown to the user. During its way out of the building, the user will be able to see virtual persons moving like in a real emergency situation where simulated user will be able to turn off and on lights and lock/unlock doors. The application will have access to a media cloud service in which videos, images, texts and presentations will be available to support the user with emergency and safety useful instructions.

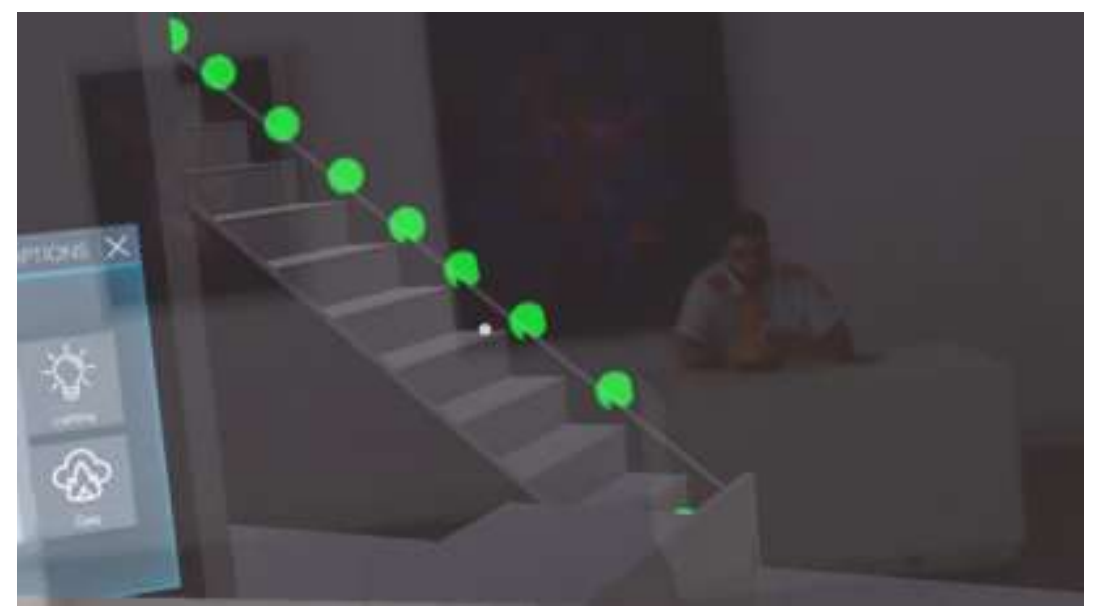

Figure 3. Escape route display in MR HoloLens $(\mathcal{C}$ Application

\section{Study Case 2 - Building VR environments used for overhead crane training operations based on shipping ports sensors information}

A good example based on an industrial environment, where virtual reality technologies can be successfully applied in order to provide highly efficient training techniques, is provided by overhead crane operations. There are many environments that are using overhead cranes: a) handling of components - where a variety of components gets cut, drilled, prepared as assembly components, b) manufacturing, for example steel factories or naval shipyards, where a bulk product is being made, that product ends up in some final form that needs to be handled by an overhead crane, d) constructions, e) machines maintenance when at certain times some machines need to be stopped and some of their parts pulled out, like motor boxes, gear boxes, rollers, parts that requires lubrication or reshaping/replacements, f) loading/unloading goods/containers. Cranes are heavy and large devices, usually around 4-5 stories tall, with a capacity around 30-ton range. Most of the cranes have 2-hook/2-hoist system, with a main hook and an auxiliary one. For example, it can be a 30-ton hook and a 5-ton hook. In heavy manufacturing or large ports, there are wider bays, 6-8 stories tall, where 50-70-ton cranes aren't uncommon. Power plants are using crane capacities having at least 125 ton, up to 300 ton, that are used for changing turbines and generator components. Nuclear and oil plants fall also in this heavy category of 125-300-ton range. Usually, overhead cranes present unique operational challenges, compared to other heavy equipment. For example, other heavy equipment can be mounted on tires or tracks, while working on ground. The operator needs to maneuver the machine and its payload by following a course with different obstacles and turns. On the other hand, overhead cranes will pick up the load and go over the obstructions and other equipment, having a flight path that is easier to arrange from take-off to set-down. But this comes with a situation where the operator has a unique and different perspective, also in a context where he is not positioned immediately near the load. The distance perspective and depth perception must be managed by the operator. Also, the timing with the machine is very important for handling the load without generating swing or some strange motion that could lead to object striking or rigging failure. There is a big difference between ground and air operations. When a ground operator sees pedestrians or some vehicles, he can manage his way around those objects. Still, for a crane operator it's not at all clear what can be found underneath their loadings. It is extremely important for a crane operator to pay attention where are the people for avoiding flights over those people. They should have abilities to manipulate their loadings over different flight path in order to avoid any possible risk. In most of the cases, ground workers don't even realize that a huge loading is above their heads, when operations are in progress. The responsibility falls on the operator, as for any worker that is using heavy machinery, to ensure that machine they are handling is not a threat for anyone or anything in its surroundings.

By using VR environments, it is possible to train crane operators in performing complex machine operations in the absence of a real crane machine. VR training processes can simulate activities and scenarios 
involving takeoff and landing points, heights, available space to manipulate the load, moving crane into specific locations for acquiring the load and performing crane operations. This can be done in shorter times than normal operations where many other activities must be performed: signaling by specialized persons, load alignment and stabilizing. For example, in a VR environment, a trainee could perform 15 tasks in an hour, whereas in a real scenario it might be able to accomplish around 3. By using virtual environments, there are economic advantages like no lost production from real cranes, no risk regarding the equipment, no obstructions risks, no danger for the people operating the crane or for other person. Also, it is possible to create situations that could test reactions in conditions like strong wind currents, failed rigging, power shortage, vehicle or pedestrian traffic. Usually, VR environments are developed in a static way, meaning that real, external conditions don't influence at all the user experience. Still, a different path could be followed. As presented in this paper, it is possible to collect data coming from various sensors deployed on different sites, in order to dynamically generate environments based on that data. Our study case describes such a scenario, developed at ALTFACTOR, where the presented architecture allows data collection from various shipping ports in order to simulate crane operations in VR environments that are taking into consideration real on-site conditions. Following this approach, any user could experience real life scenarios based on precise conditions that are happening in ports that could be located thousands of miles away. Figure 4 presents the architecture that should be able to sustain developing this kind of environments.

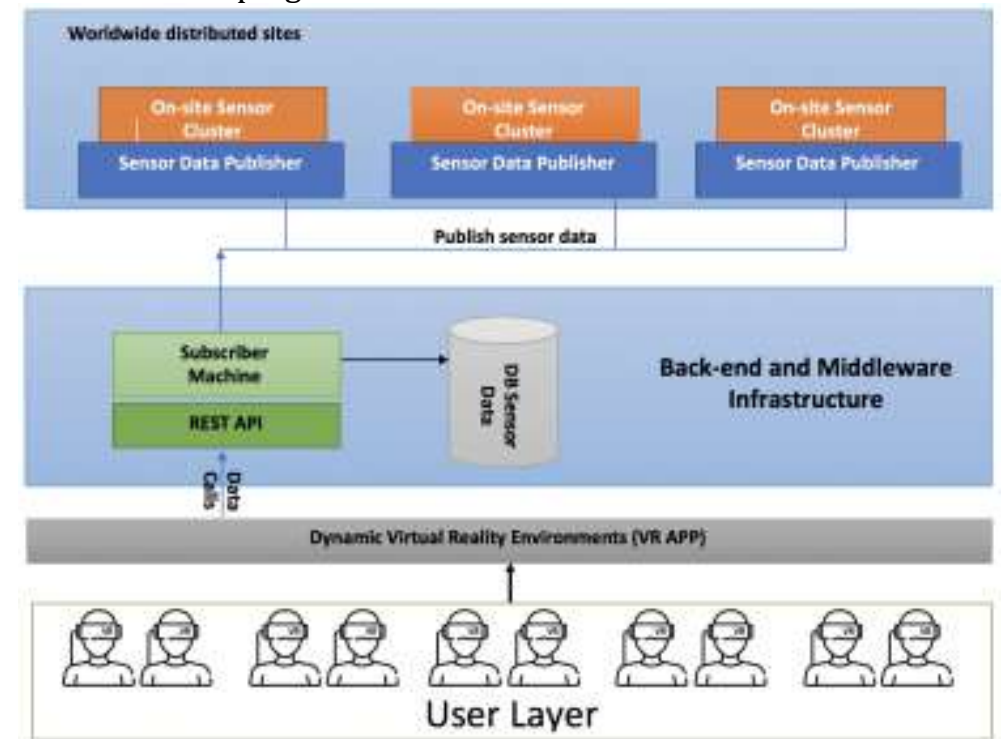

Figure 4. Architectural overview on how to develop dynamic VR environments based on real sensor data

As figure 4 presents, data coming from sensor clusters deployed in different shipping ports can be transmitted via through a publish-subscribe architecture to any party interested in collecting that data. In our case, a subscriber machine will subscribe to specific topics, having the semantics needed to build the VR environment and it will store data coming from various port sites.

The sensor data stored in the database is made available through a REST API to the VR Unity software app that will prepare the environment based on the data it is receiving.

At the moment, the sensors that were taken into consideration are related to the following aspects: inside crane temperature, outside crane temperature, wind speed at 15 and 30 meters, wind direction, rain intensity, luminosity and visibility, with the possibility of extending to on-site human presence/detection. These sensors where chose due to the importance of weather when performing crane operations.

Various weather conditions can affect stability, visibility and workers' performance. It is well known that people working with cranes are continuously exposed to harsh environments while working in rain, scorching heat, or dense fog situations. When we are talking about big ports, where automated processes cannot undertake $100 \%$ of the tasks, the workers will have to perform their best, even if risks are high.

We developed an environment that will help build good skills in an overhead crane operator. It allows evaluation of the controls and, in case of a malfunction, the operator should take decisions on how to solve the issue. Operator activities can be developed as for operator-only involvement or with some integration with signal or tagline persons.

Crane manipulation involves loading items, turn/up-righting and down-righting activities, which usually requires skill and time to obtain proper load reactions. Flying items, long tall items or nearby people requires a very good depth perception. Also, some scenarios can be developed for determining accuracy and speed, where time is a sensitive component. For example, extreme heat conditions, heavy winds, poor 
visibility due to night or fog conditions, cryogening tanks manipulation requires both short times of manipulation and extreme precision.

Besides time and precision, VR techniques can reproduce types of actions that requires concentration, like checking if the crane hook is arriving quietly, without swing. As mentioned before, the environments defined in the application will take into consideration the data sent by the on-site sensors. The user will choose a site where he wants to train and the first version of the environment will be created accordingly to the real on-site conditions in terms of wind strength, light intensity, heavy/light rain or no rain at all, real temperatures, etc. The crane monitor will display the specified conditions. Of course, in case user wants to add or modify the initial conditions he is free to do this. For example, figure 5 presents a scenario where the conditions are clear and quite sunny, with people present below the transported items.
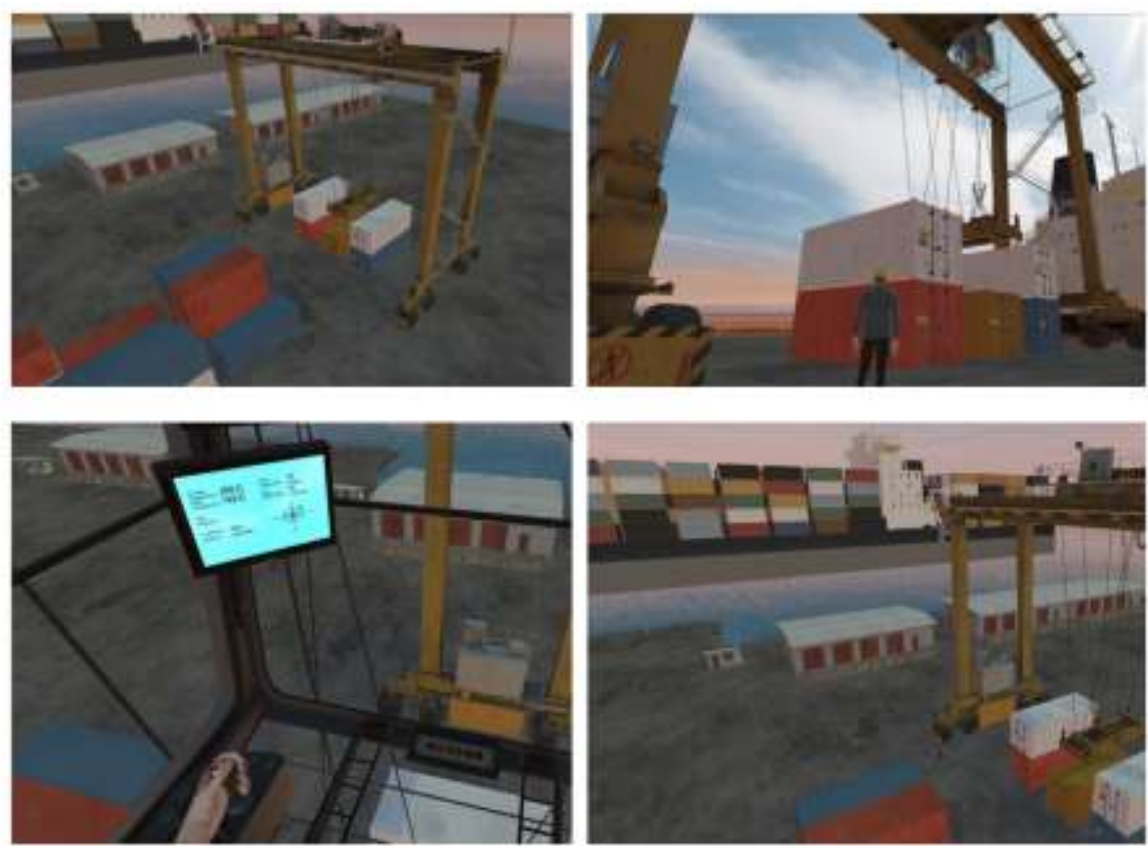

Figure 5. Crane VR environment in a sunny day

Each moment, the user can see, displayed on the VR monitor, real external conditions coming directly from the site he chose to train on, see figure 6:

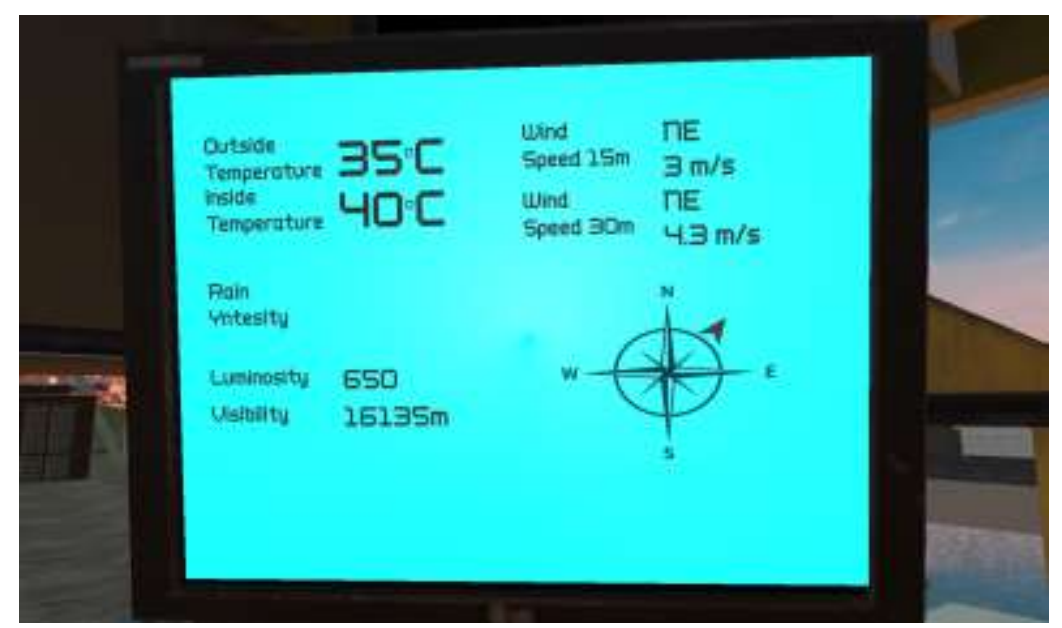

Figure 6. VR crane monitor with real sensor information (sunny day)

If weather conditions changes, the virtual environment also changes. The figures below, captured from the VR application, presents such a scenario where the incoming data is indicating heavy rain/winds and low visibility, with human presence under the transported item. 

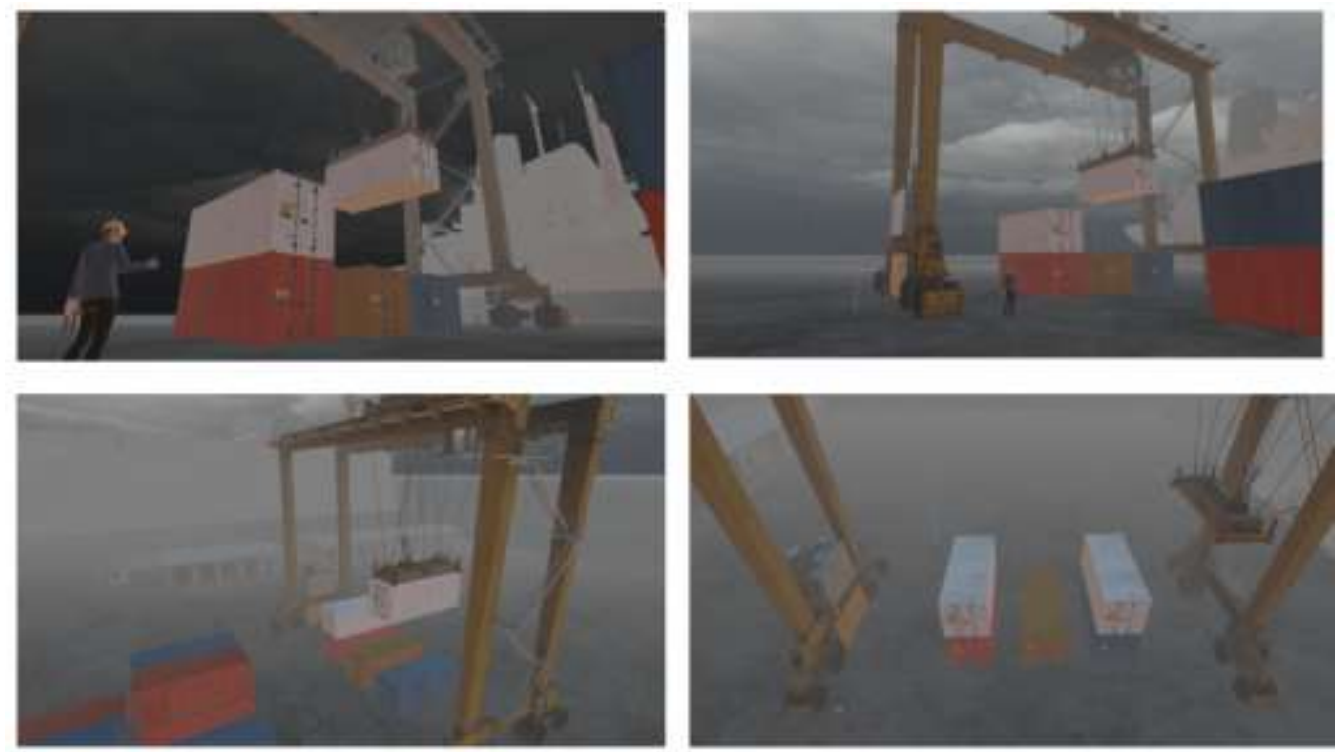

Figure 7. Crane VR environment for a rainy day

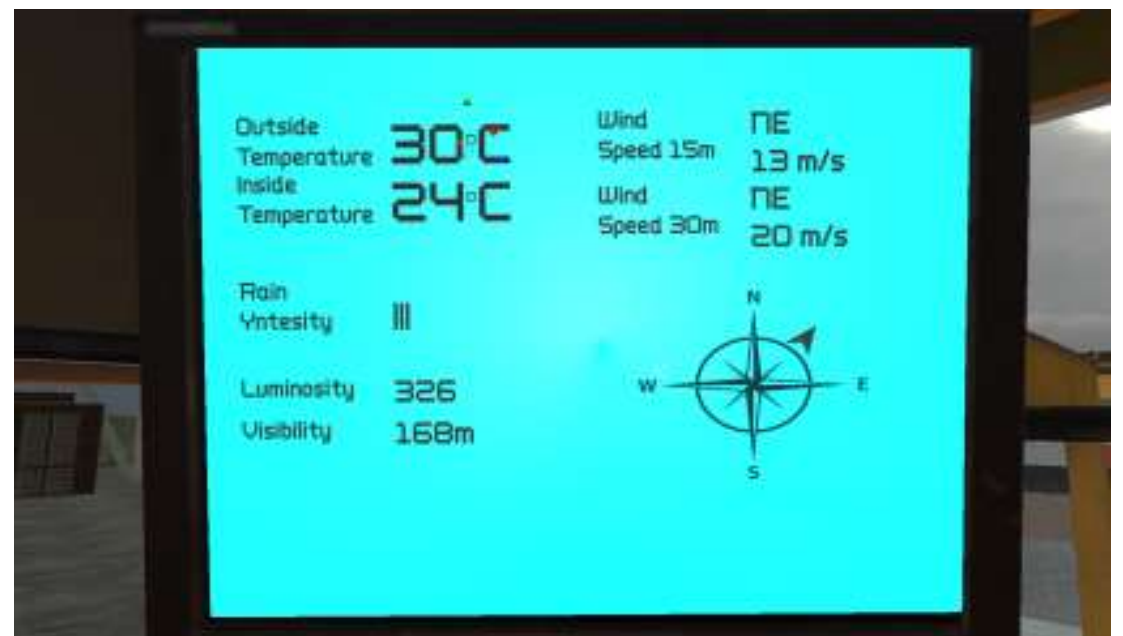

Figure 8. VR crane monitor with real sensor information (rainy day)

\section{Conclusion}

Under CITISIM project in the field of smart cities, MR/VR services have a great potential. In this work we have presented how to inject IoT information coming from the real world to the virtual world used in MR/VR services. In the first study case an emergency evacuation service is presented, the service can simulate evacuation paths at real-time to choose the most appropriate one and present to the user together with information coming from IoT architecture (e.g. smoke sensors) to help the user to choose the most appropriate path and to anticipate the state of the route where he is going to be evacuated. Second study case presents a developed VR environment that will help training the overhead crane operators. The presented virtual reality application allows the evaluation of the controls and it can be possible to dynamically build the environments based on incoming IoT information from many sites (shipping ports). As such, real weather conditions like extreme heat, heavy winds, poor visibility due to night or fog conditions can influence the rendered scene. In both uses cases, the information coming from IoT domain supports the decision/training process showing the big impact that this mix of technologies can provide.

\section{Acknowledgments}

Two cases of use for smart cities management has been presented in this paper that presents how Mixed Reality and Virtual Reality can simulate different scenarios providing the capability of minimize the time of response. The presented use cases are based on CitiSim smart city platform, which incorporates a series of intelligent services based on emerging technologies. CitiSim has not been developed only for the presented use-cases. Its final objective is to act as a digital twin of the city and to be a digital-virtual representation as easy to manage as possible in the city, that serves as a simulation, monitoring and management tool for the prediction of crises, emergency situations or for improving citizen wellbeing. CitiSim is a European R \& D project, with ITEA3 seal, funded by MINETAD, Subprogram: Strategic Action Economy and Digital Society (AEESD) and by CDTi, through INNOGLOBAL support. 


\section{References}

1. Dávideková, M.; Mjartan, M.; Greguš, M (2017). Utilization of virtual reality in education of employees in slovakia. Procedia Comput. Sci, 113, 253-260.

2. Davis B., Bryla K (2015). Benton, Oculus Rift in Action. Manning Publications Co.

3. Dominic, M.; Margaret,W.; Timothy, J.M.; Craig, S.; Michael,W (2016).. Student experiences of virtual reality: A case study in learning special relativity. Am. J. Phys., 78, 862-868.

4. Elby, R.; Mahmoud, M.B.; Roy, G (2017). The need for virtual reality simulators in dental education: A review. Saudi Dent. J. Apr; 29(2), 41-47.

5. Federica, P.; Nicola, T.; Luca, A.; Luciana, A.; Fabrizia, M (2015). Developing effective virtual reality training for military forces and emergency operators: From technology to human factors. In Proceedings of the 14th International Conference on Modeling and Applied Simulation, Seattle,WA, USA, 14-16, 176-187.

6. Hale K.S. and Stanney K.M. (2014), Handbook of Virtual Environments: Design, Implementation, and Applications, 2nd ed., K. S. Hale and K. M. Stanney, Eds. CRC Press.

7. Huang, Y.; Ali, S.; Bi, X.; Zhai, X.; Liu, R.; Guo, F.; Yu, P (2016). Research on Smart Campus Based on the Internet of Things and Virtual Reality. Int. J. Smart Home, 10, 213-220.

8. Jerald J. (2015), The VR Book: Human-Centered Design for Virtual Reality. Morgan \& Claypool publishers and ACM Books

9. Liu, D.; Valdiviezo-Díaz, P.; Riofrio, G.; Sun, Y.-M.; Barba, R (2015). Integration of Virtual Labs into Science E-learning. In Proceedings of the International Conference on Virtual and Augmented Reality in Education, Monterrey, Mexico, 19-21 November; pp. 95-102.

10. Muhanna M.A. (2015). "Virtual reality and the cave: Taxonomy, interaction challenges and research directions," Journal of King Saud University - Computer and Information Sciences, 27(3), 344-361

11. Numan, A.; Sehat, U.; Aftab, A.; Jamal, R (2014). Interactive Virtual Chemistry Laboratory for Simulation of High School Experiments. In Proceedings of the International Conference on Computer Graphics, Animation and Gaming Technologies, Ankara, Turkey.

12. Parisi T. (2015). Learning Virtual Reality - Developing Immersive Experiences and Applications for Desktop, Web, and Mobile. O'Reilly Media.

13. Swan, $M$ (2012). Sensor mania! the internet of things, wearable computing, objective metrics, and the quantified self 2.0. J. Sens. Actuator Netw, 1, 217-253.

14. Talari, S.; Shafie-Khah, M.; Siano, P.; Loia, V.; Tommasetti, A.; Catalão, J (2017). A review of smart cities based on the internet of things concept, 10, 421.

15. Vangelista, L.; Zanella, A.; Zorzi, M (2015). Long-range IoT technologies: The dawn of LoRaTM. In Future Access Enablers of Ubiquitous and Intelligent Infrastructures; Springer: Cham, Switzerland, 51-58.

16. Vaughan, N.; Dubey, V.N.; Wainwright, T.W.; Middleton, R.G (2015). A review of virtual reality based training simulators for orthopaedicsurgery. Med. Eng. Phys, 38, 1-13.

17. Veeramanickam, M.R.M.; Mohanapriya, M (2016). IoT enabled Futurus Smart Campus with effective E-Learning: I-Campus. GSTF J. Eng. Technol, 3, 81-87

18. Wu, J.-W.; Chou, D.-W.; Jiang, J.-R (2014). The Virtual Environment of Things (VEoT): A Framework for Integrating Smart Things into Networked Virtual Environments. In Proceedings of the IEEE International Conference on Internet of Things (iThings), and IEEE Green Computing and Communications (GreenCom) and IEEE Cyber, Physical and Social Computing (CPSCom), Taipei, Taiwan, 1-3 September, pp. 456-459.

19. https://appreal-vr.com/blog/vr-and-internet-of-things/

20. Emerging Technologies: Changing how we live, work and play EY-Mint Emerging Technologies Report 2019.

21. What Is Mixed Reality And What Does It Mean for Enterprise?https://www.forbes.com/sites/solrogers/2018/12/04/what-is-mixedreality-and-what-does-it-mean-for-enterprise/\#285ea8445df9 